\title{
Connaissances, Attitudes Et Pratiques En Matiere De Cancer Du Col De L'uterus (Ccu) Chez Les Professionnels De Sante A Parakou Au Benin En 2016
}

\section{Achille Awadé Afoukou Obossou}

UER en gynécologie Obstétrique, Faculté de Médecine, Université de Parakou, Bénin

\section{Moufalilou Aboubakar \\ Mathieu Ogoudjobi}

UER en Gynécologie Obstétrique, Faculté des sciences de la Santé,

Université d'Abomey Calavi, Bénin

Sedjro Raoul Atade

Mahublo Vinadou Vodouhe

Rachidi Imorou Sidi

Nicephore Dagan

Fanny Maryline Nouessèwa Hounkponou Ahouingnan

Kabibou Salifou

UER en gynécologie Obstétrique, Faculté de Médecine, Université de Parakou, Bénin

\section{Perrin René Xavier}

UER en Gynécologie Obstétrique, Faculté des sciences de la Santé, Université d'Abomey Calavi, Bénin

\section{Doi:10.19044/esj.2021.v17n25p290}

Submitted: 28 January 2021

Accepted: 22 June 2021

Published: 31 July 2021
Copyright 2021 Author(s)

Under Creative Commons BY-NC-ND 4.0 OPEN ACCESS

Cite As:

Obossou. A.A.A., Aboubakar M., Ogoudjobi M., Atade S.R., Vodouhe M.V., Dagan N., Hounkponou Ahouingnan F.M.N., Salifou K. \& Perrin R.X. (2021). Connaissances, Attitudes Et Pratiques En Matiere De Cancer Du Col De L'uterus (Ccu) Chez Les Professionnels De Sante A Parakou Au Benin En 2016. European Scientific Journal, ESJ, 17(25), 290.

https://doi.org/10.19044/esj.2021.v17n25p290

\section{Résumé}

Introduction: En Afrique et dans les pays en développement, le cancer du col de l’utérus représente le 1er cancer chez la femme. 
Objectifs: Evaluer les connaissances, attitudes et pratiques des professionnels de santé en matière de cancer du col de l'utérus à Parakou en 2016.

Cadre et méthode d'étude: Il s'est agi d'une étude transversale descriptive de type connaissance, attitude et pratique (CAP) chez les agents de santé de la ville de Parakou au Nord du Bénin. Elle a couvert une période de 3 mois allant du 1ermars 2016 au 1er juin 2016.

Résultats: Au total 133 agents de santé avaient participé à l'étude. L'âge moyen était de 36 ans \pm 8 ans avec des extrêmes de 19 ans et 70 ans. Les facteurs de risque étaient connus par 1,5\% de la population d'étude. Soixante-six virgule deux pour cent $(66,2 \%)$ des enquêtés méconnaissaient l'existence de vaccins contre le HPV. La connaissance sur le cancer du col de l'utérus était $71,4 \%$. Pour 98,5\% le cancer du col de l'utérus est une affection grave et $81,2 \%$ trouvent que le dépistage de ce cancer est mal organisé au Bénin. De façon globale, 27,8\% des agents avaient une attitude juste en matière de cancer du col de l'utérus. Soixante-dix-huit virgule neuf pour cent (78,9\%) des enquêtés pratiquaient le dépistage de ce cancer. Parmi eux 71,4\% pratiquent l'IVA/IVL et 14,3\% le FCV mais seulement 1,5\% des enquêtés avaient une pratique adéquate face au cancer du col de l'utérus. Au total 5 agents sur les 133 (3,8\%) avaient un bon score CAP global.

Conclusion: Ces résultats mettent en évidence la nécessité d'une formation continue des professionnels de santé y compris les paramédicaux en matière du cancer du col.

Mots clés : Cancer Du Col De L’utérus, Connaissance, Attitudes, Pratiques 


\section{Knowledge, Attitudes And Practices On Cervical Cancer (Cc) Of Health Care Providers In Parakou, Benin In 2016}

\section{Achille Awadé Afoukou Obossou}

UER en gynécologie Obstétrique, Faculté de Médecine, Université de Parakou, Bénin

\section{Moufalilou Aboubakar \\ Mathieu Ogoudjobi}

UER en Gynécologie Obstétrique, Faculté des sciences de la Santé, Université d’Abomey Calavi, Bénin

Sedjro Raoul Atade

Mahublo Vinadou Vodouhe

Rachidi Imorou Sidi

Nicephore Dagan

Fanny Maryline Nouessèwa Hounkponou Ahouingnan

Kabibou Salifou

UER en gynécologie Obstétrique, Faculté de Médecine, Université de Parakou, Bénin

\section{Perrin René Xavier}

UER en Gynécologie Obstétrique, Faculté des sciences de la Santé, Université d’Abomey Calavi, Bénin

\section{Abstract}

Introduction: in Africa and developing countries, cervical cancer is the leading cancer in women.

Objectives: Assess knowledge, attitudes and practices of health care professionals as regards cervical cancer in Parakou in 2016.

Framework and methodology: This was a descriptive cross-sectional knowledge, attitude and practice (KAP) study among health workers in the city of Parakou in northern Benin. It covered a period of 3 months from March 1, 2016 to June 1, 2016.

Results: In total 133 health workers were involved in the survey. The average age was 36 years \pm 8 years with extremes of 19 years and 70 years. The risk factors were known by $1.5 \%$ of the study population. Sixty-six-point two percent (66.2\%) of respondents were unaware of the existence of HPV vaccines. On the whole, they had average knowledge of cervical cancer (71.4\%). For 98.5\% cervical cancer is a severe disease and $81.2 \%$ think that the screening of this cancer is poorly organized in Benin. Overall, only $27.8 \%$ of the workers had a right attitude as regards cervical cancer. Seventy-eightpoint nine percent (78.9\%) of respondents practiced screening for this cancer; $71.4 \%$ among them performed VIA/ VILI and 14.3\% cervical smear but only 
$1.5 \%$ of the surveyed had a suitable attitude as regards cervical cancer. In total, only 5 workers out of 133 (3.8\%) had a good overall KAP score.

Conclusion: These results bring to the fore the need for continuing training for healthcare professionals including paramedics as regards cervical cancer.

Key words: Cervical Cancer, Knowledge, Attitudes, Practices

\section{Introduction}

Le cancer du col de l'utérus est un processus malin lié à l'infection persistante par certains papillomavirus humains (HPV) dits oncogènes. Il vient au deuxième rang des cancers féminins dans le monde ; premier cancer en Afrique et deuxième cancer après le cancer du sein chez la femme en Europe (Baldauf et al, 2010). Ce cancer est accessible au dépistage, ce qui améliore son pronostic de guérison lorsqu'il est dépisté tôt. Il touche tous les ans dans le monde près de 500000 femmes dont $80 \%$ dans les pays en développement (Lansac et al, 2014

Dans les pays en développement, $80 \%$ des cas de cancer du col ne sont pas diagnostiqués ou sont déjà incurables au moment du diagnostic. Les campagnes de dépistage du cancer du col mises sur pied dans certains pays en développement n'ont pas contribué à faire baisser l'incidence ou la mortalité liée à ce cancer. On estime que, au niveau mondial, une femme meurt du cancer de l'utérus toutes les deux minutes et 8 de ces cancers sur 10 ont lieu dans des pays en voie de développement (OMS,2008; Isautier,2012)

Au Bénin la mortalité est de 14,3 pour 100000 habitants pour une incidence de 21,5 pour 100000 habitants (OMS, 2008). la fréquence reste élevée en partie parceque le personnel médical ne propose pas systématiquement le dépistage. Ces cinq dernières années au centre national hospitalier et universitaire Hubert Koutoucou MAGA (CNHU-HKM) de Cotonou le cancer du col de l'utérus venait au deuxième rang des cancers féminins avec 16,8\% après celui du sein 32,5\% (Tonato Bagnan et al, 2013). Dans une étude rétrospective menée à l'Hôpital de la Mère et de l'Enfant Lagune (HOMEL) et à la clinique Universitaire de Gynécologie Obstétrique (CUGO) de Cotonou en 2013 le cancer du sein venait au premier rang avec $44,3 \%$ des cas et le cancer du col représente $26,7 \%$ des cas. De cette enquête, il ressort que le dépistage des cancers gynécologiques n’est pas bien organisé dans notre pays ainsi que l'éducation à la santé et à la reproduction de la femme (Tonato Bagnan et al, 2013 ; Ministère santé 2014)

Dans une étude réalisée en Ouganda en 2006 (Twaha et al, 2006), 29\% des médecins généralistes avaient reconnu le HPV comme principal facteur de risque du cancer du col de l'utérus. Dans une autre étude réalisée au Maroc en 2013 chez des médecins généralistes, seulement 21\% parmi eux avaient 
identifié le HPV comme facteur de risque et 92,8\% n'avaient jamais pratiqué de FCV chez leurs patientes (Nani et al,2013). Ceci montre que le niveau de connaissance de ces médecins était relativement faible. Par contre à Cotonou au Bénin en 2001, une étude avait noté un bon niveau de connaissance des agents de santé concernant les moyens de dépistage $(98,4 \%)$ et les moyens thérapeutiques $(87,1 \%)$ du cancer du col de l'utérus (CCU)(Komongui et $\mathrm{al}, 2000)$.

L'importance du rôle du personnel soignant dans le dépistage du cancer du col a été largement démontrée par plusieurs auteurs. En raison de leur place centrale dans le système de soin et de leur rôle primordial en matière d'information et d'orientation des patients; les personnels soignants peuvent contribuer efficacement à la sensibilisation, à l'information et à la motivation des femmes pour du dépistage. C’est ce qui a motivé le présent travail dont l'objectif vise à étudier les Connaissances, Attitudes et Pratiques en matière de cancer du col de l'utérus chez les professionnels de la santé en matière de cancer du col de l'utérus à Parakou en 2016.

\section{Cadre et méthode}

Notre étude a eu pour cadre les différentes formations sanitaires publiques et privées de la ville de Parakou, la plus grande ville du Nord BENIN.

Il s'était agit d'une étude transversale à visée descriptive et analytique avec collecte prospective des données. L'étude s'est déroulée sur une période de 3 mois allant du $1^{\mathrm{er}}$ mars 2016 au $1^{\mathrm{er}}$ juin 2016. La population d'étude était constituée de l'ensemble des professionnels de santé de la ville de Parakou.

Ont été inclus: les professionnels de santé (gynécologues, médecins généralistes, sages-femmes, infirmiers) exerçant dans les formations sanitaires publiques et privées, intervenant dans les activités de consultations prénatales, postnatales, de gynécologie ou de planning familial, présents sur les sites les jours d'enquête et ayant donné leur consentement.

Les professionnels de santé non consentants pour participer à l'étude et ceux absents les jours de la collecte des données n'ont pas été inclus.

Nous avons procédé à un recensement exhaustif des cibles répondant aux critères de sélection. Les connaissances, attitudes et pratiques des professionnels de santé étaient les variables dépendantes. En s'inspirant du modèle d'Essi et al(2013) l'évaluation du niveau de connaissance a été quantifiée. Il a été restitué en 4 niveaux (mauvais, insuffisant, moyen et bon).

- Moins de $50 \%$ de bonnes réponses = Mauvais

- Entre $50 \%$ et $65 \%$ de bonnes réponses = Insuffisant

- Entre $65 \%$ et $85 \%$ de bonnes réponses = Moyen

- Plus de $85 \%$ de bonnes réponses = Bon 
Pour ce qui concerne l'attitude, la grille d'analyse a mis l'accent sur 4 critères (juste, approximatif, erroné et néfaste).

- $\quad$ Moins de $50 \%$ de bonnes réponses = néfaste

- Entre $50 \%$ et $65 \%$ de bonnes réponses = erroné

- Entre $65 \%$ et $85 \%$ de bonnes réponses = approximatif

- Plus de $85 \%$ de bonnes réponses = juste adéquat).

L'analyse de la pratique a été établie en 3 niveaux (néfaste, inadéquat,

- Moins de $50 \%$ de bonnes réponses = néfaste

- Entre $50 \%$ et $85 \%$ de bonnes réponses = inadéquat

- Plus de $85 \%$ de bonnes réponses = adéquat

Les données recueillies ont été analysées avec le logiciel SPSS, version 17.0.

Les variables quantitatives ont été exprimées en moyennes avec un écart type et les variables qualitatives en pourcentages simple. Le test statistique $\mathrm{Chi}^{2}$ de Pearson ou de Fisher a été utilisé selon le cas pour la comparaison des données qualitatives. La différence a été considérée comme statistiquement significative pour une probabilité $\mathrm{P}<0,05$.

Les consentements écrits des enquêtés ont été obtenu.

\section{Resultats}

À l'issue de notre enquête menée auprès des professionnels de santé de la ville de Parakou sur le cancer du col de l'utérus, 133 agents sur un total de 145 ont participé à l'enquête soit un taux de participation de 91,72\%.

\section{Caractéristiques sociodémographiques de la population d'étude}

L’âge moyen était de $36 \pm 8$ ans avec des extrêmes de 19 et 70 ans. La tranche d'âge la plus représentée était celle de 25 ans à 34 ans (41,4\%).

Les sujets de sexe féminin représentaient 79,7 \%; soit une sex-ratio de 0,25 (25 hommes pour 100 femmes).

La répartition par âge est représentée sur la figure numéro 1.

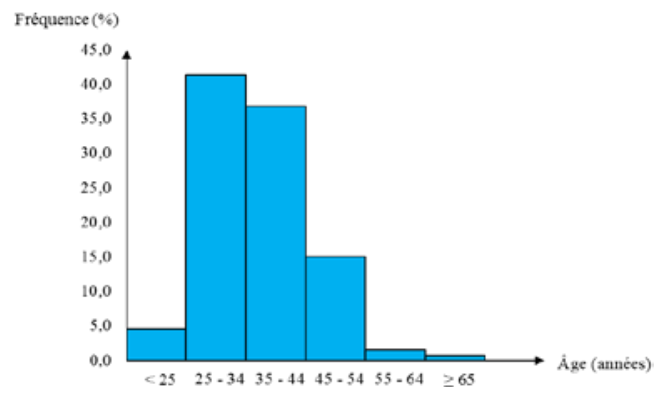

Figure 1. Répartition des agents de santé sondés sur le cancer du col selon l'âge dans les formations sanitaires publiques et privées de Parakou en $2016(\mathrm{~N}=133)$ 


\section{Connaissance sur le cancer du col de l'utérus Description des facteurs de risque}

Les facteurs de risque du cancer du col de l'utérus étaient connus par 1,5\%. Cependant (39,8\%) d'entre eux ont cité le HPV comme facteur de risque principal. Ces informations sont représentées dans le tableau I.

Tableau I. Répartition des agents de santé sondés sur le cancer du col selon le niveau de connaissance des facteurs de risque du cancer du col de l'utérus dans les formations sanitaires publiques et privées de Parakou en $2016(\mathrm{~N}=133)$

\begin{tabular}{|l|c|c|}
\hline & Effectif & Fréquence (\%) \\
\hline \multicolumn{3}{|c|}{ Facteurs de risque } \\
\hline Bon & 2 & 1,5 \\
\hline Moyen & 57 & 42,9 \\
\hline Insuffisant & 55 & 41,4 \\
\hline Mauvais & 19 & 14,2 \\
\hline Total & 133 & 100,0 \\
\hline \multicolumn{3}{|c|}{ Facteur de risque principal (HPV) } \\
\hline Oui & 53 & 39,8 \\
\hline Non & 80 & 60,2 \\
\hline Total & 133 & 100,0 \\
\hline
\end{tabular}

\section{Existence de vaccin et moyens de dépistage}

Plus de la moitié (66,2\%) des agents sondés ignoraient l'existence d'un vaccin contre le cancer du col et seuls 6,8\% connaissaient bien les moyens de dépistage. Ces informations sont représentées sur le tableau II.

\begin{tabular}{|l|c|c|}
\hline \multicolumn{1}{|c|}{ Existence d'un Vaccin } & Effectif & Fréquence (\%) \\
\hline Oui & & 33,8 \\
\hline Non & 45 & 66,2 \\
\hline Total Moyens de dépistage & 88 & $\mathbf{1 0 0 , 0}$ \\
\hline \multicolumn{1}{|c|}{$\mathbf{1 3 3}$} & 6,8 \\
\hline Bon & 9 & 19,5 \\
\hline Moyen & 26 & 68,4 \\
\hline Insuffisant & 91 & 5,3 \\
\hline Mauvais & 7 & $\mathbf{1 0 0 , 0}$ \\
\hline Total & $\mathbf{1 3 3}$ & \\
\hline
\end{tabular}

Tableau II. Répartition des agents de santé sondés sur le cancer du col selon le niveau de connaissance des moyens de dépistage du cancer du col de l'utérus et l'existence de vaccin dans les formations sanitaires publiques et privées de Parakou en $2016(\mathrm{~N}=133)$

\section{Signes évocateurs, examens paracliniques, méthodes thérapeutiques}

Les agents de santé avaient un niveau moyen de connaissance des signes cliniques (57,9\%), des examens paracliniques à prescrire (61.7\%) et des méthodes thérapeutiques $(40,6 \%)$. Ces informations se retrouvent dans le tableau III. 
Tableau III. Répartition des agents de santé sondés sur le cancer du col selon le niveau de connaissance des signes évocateurs, les examens paracliniques à prescrire et les méthodes thérapeutiques du cancer du col de l'utérus dans les formations sanitaires publiques et privées de Parakou en $2016(\mathrm{~N}=133)$

\begin{tabular}{|c|c|c|}
\hline & Effectif & Fréquence (\%) \\
\hline Signe évocateur & & 38,3 \\
Bon & 51 & 57,9 \\
\hline Moyen & 77 & 3,0 \\
\hline Insuffisant & 4 & 0,8 \\
\hline Mauvais & 1 & $\mathbf{1 0 0 , 0}$ \\
\hline Total & $\mathbf{1 3 3}$ & 11,3 \\
\hline Examen paraclinique & & 61,7 \\
\hline Bon & 15 & 18,8 \\
\hline Moyen & 82 & 8,2 \\
\hline Insuffisant & 25 & $\mathbf{1 0 0 , 0}$ \\
\hline Mauvais & 11 & 3,8 \\
\hline Total & $\mathbf{1 3 3}$ & 40,6 \\
\hline Méthode thérapeutique & & 40,6 \\
\hline Bon & 5 & 15,0 \\
\hline Moyen & 54 & $\mathbf{1 0 0 , 0}$ \\
\hline Insuffisant & 54 & 20 \\
\hline Tauvais & $\mathbf{1 3 3}$ & \\
\hline & & \\
\hline & & \\
\hline & & \\
\hline
\end{tabular}

\section{Niveau de connaissance globale}

\section{Connaissance subjective}

Sur l'ensemble des agents de santé, 123 (92,5\%) ont répondu avoir connaissance du cancer du col de l'utérus.

\section{Connaissance objective}

Les agents ayant un niveau de connaissance globale moyen représentaient $71,4 \%$. Ce niveau était bon chez 1,5\%. Le niveau de connaissance des agents est schématisé sur la figure 2 .

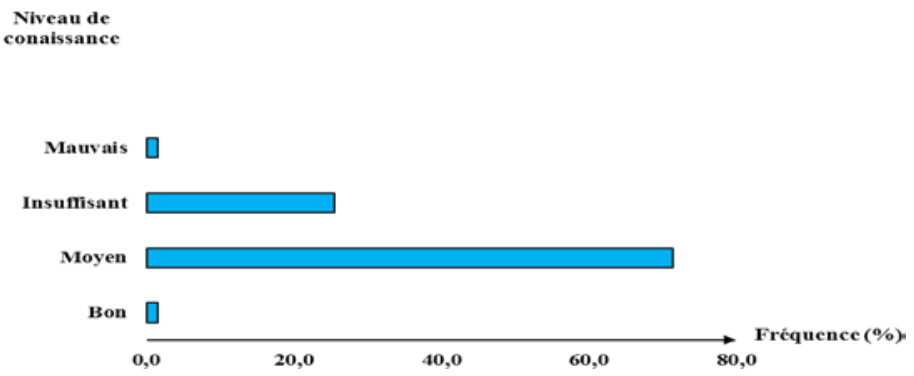

Figure 2. Répartition des agents de santé sondés sur le cancer du col selon leur niveau de connaissance globale à l'aide du score CAP d'ESSI dans les formations sanitaires publiques et privées de Parakou en $2016(\mathrm{~N}=133)$ 


\section{Attitudes, Perceptions en matière de cancer du col de l'utérus}

Perception sur la gravité, la conduite du dépistage et le caractère curable

Le cancer du col utérin était perçu comme une affection grave par 98,5\% et 81,2\% avaient jugé le dépistage mal mené au Bénin.

Quant à la curabilité de cette maladie 92,5\% l'avait déclarée possible si le diagnostic est précoce (123 agents) ou la prise en charge adéquate (113 agents). Ces informations se retrouvent dans le tableau IV

Tableau IV. Répartition des agents de santé sondés sur le cancer du col selon leur perception sur la gravité, la conduite du dépistage et la curabilité du cancer du col de l'utérus dans les formations sanitaires publiques et privées de Parakou en $2016(\mathrm{~N}=133)$

\begin{tabular}{|c|c|c|}
\hline & Effectif & Fréquence (\%) \\
\hline Gravité & & \\
\hline Oui & 131 & 98,5 \\
\hline Non & 2 & 1,5 \\
\hline Total & $\mathbf{1 3 3}$ & $\mathbf{1 0 0 , 0}$ \\
\hline Conduite dépistage & & \\
\hline Bien organisée & 5 & 3,8 \\
\hline Mal organisée & 108 & 81,2 \\
\hline Inexistante & 7 & 5,2 \\
\hline Indifférent & 13 & 9,8 \\
\hline Total & $\mathbf{1 3 3}$ & $\mathbf{1 0 0 , 0}$ \\
\hline Curabilité du cancer & & \\
\hline Oui & 123 & 92,5 \\
\hline Non & 10 & 7,5 \\
\hline Total & $\mathbf{1 3 3}$ & $\mathbf{1 0 0 , 0}$ \\
\hline & & \\
\hline
\end{tabular}

\section{Attitude face au dépistage systématique}

La quasi-totalité $(94,7 \%)$ des agents était favorable au dépistage systématique du CCU soit par la technique de l'IVA/IVL (85,7\%) soit par le frottis cervico vaginal FCV) (63,2\%). Ces informations sont représentées dans le tableau $\mathrm{V}$

Tableau V. Répartition des agents de santé sondés sur le cancer du col selon leur attitude face au dépistage du cancer du col de l'utérus dans les formations sanitaires publiques et privées de Parakou en $2016(\mathrm{~N}=133)$

\begin{tabular}{|c|c|c|}
\hline & Effectif & Fréquence (\%) \\
\hline Dépistage systématique & & 94,7 \\
\hline Favorable & 126 & 5,3 \\
\hline Non favorable & 7 & $\mathbf{1 0 0 , 0}$ \\
\hline Total & $\mathbf{1 3 3}$ & \\
\hline IVA/IVL & & 85,7 \\
\hline Favorable & 114 & 14,3 \\
\hline Non favorable & 19 & $\mathbf{1 0 0 , 0}$ \\
\hline Total & $\mathbf{1 3 3}$ & 63,2 \\
\hline Frottis cervico-vaginal & & \\
\hline Favorable & 84 & \\
\hline
\end{tabular}




\begin{tabular}{|c|c|c|}
\hline Non favorable & 49 & 36,8 \\
\hline Total & $\mathbf{1 3 3}$ & $\mathbf{1 0 0 , 0}$ \\
\hline
\end{tabular}

\section{Attitude globale des agents}

De façon globale $64,7 \%$ des agents ont eu une attitude «approximative » et $27,8 \%$ une attitude «juste ». Ces résultats sont représentés sur la figure $n^{\circ} 3$.

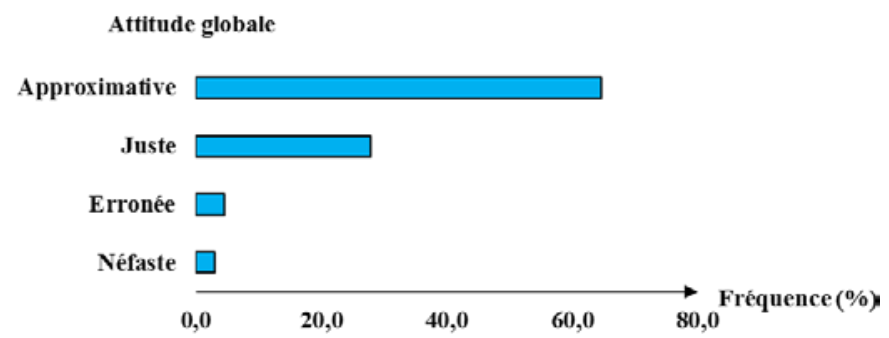

Figure 3. Répartition des agents de santé sondés sur le cancer du col selon leur attitude globale dans les formations sanitaires publiques et privées de Parakou en 2016 ( $\mathrm{N}=133)$

\section{Pratique du dépistage du cancer du col de l'utérus}

\section{Formation initiale sur le dépistage}

Une formation initiale sur le dépistage du CCU a été reçue par 83(62,4\%) agents.

\section{Tests utilisés pour la pratique du dépistage aux patientes}

Pour les 105 agents de santé $(78,9 \%)$ qui ont pratiqué un test de dépistage du CCU à leur patiente les moyens utilisés étaient l'IVA\IVL $(71,4 \%)$ et le FCV $(14,3 \%)$.

Ces informations sont représentées dans le tableau VI.

Tableau VI. Répartition des agents de santé selon les moyens utilisés pour le dépistage du CCU $(n=105)$

\begin{tabular}{|c|c|c|}
\hline & Effectif & Fréquence (\%) \\
\hline IVA/IVL & & \\
\hline Oui & 75 & 71,4 \\
\hline Non & 30 & 28,6 \\
\hline Total & 105 & 100,0 \\
\hline Fottis & & \\
\hline Oui & 15 & 14,3 \\
\hline Non & 90 & 85,7 \\
\hline Total & 105 & 100,0 \\
\hline
\end{tabular}

\section{Pratique personnelle des enquêtées face au frottis cervico-vaginal}

Parmi les 106 sujets de sexe féminin, 38 (35,8\%) ont bénéficié une fois du frottis cervico-vaginal. Seuls 6 (22,2\%) sujets de sexe masculin ont fait 
bénéficier du FCV à leur épouse. Ces résultats sont représentés dans le tableau VII.

Tableau VII. Répartition des agents de santé sondés sur le cancer du col selon leur pratique personnelle du FCV dans les formations sanitaires publiques et privées de Parakou en 2016

\begin{tabular}{|c|c|c|}
\hline & Effectif & Fréquence (\%) \\
\hline Sujets femmes & & \\
\hline Oui & 38 & 35,8 \\
\hline Non & 68 & 64,2 \\
\hline Total & $\mathbf{1 0 6}$ & $\mathbf{1 0 0 , 0}$ \\
\hline Epouse sujet homme & & 22,2 \\
\hline Oui & 6 & 77,8 \\
\hline Non & 21 & $\mathbf{1 0 0 , 0}$ \\
\hline Total & $\mathbf{2 7}$ & \\
\hline
\end{tabular}

\section{Pratique globale des agents}

Les agents de santé qui avaient une pratique « inappropriée » face au dépistage des lésions précancéreuses du col utérin (CCU) représentaient $69,9 \%$ contre $1,5 \%$ chez qui la pratique a été jugée "adéquate ». Ces informations sont représentées sur la figure $n^{\circ} 4$.

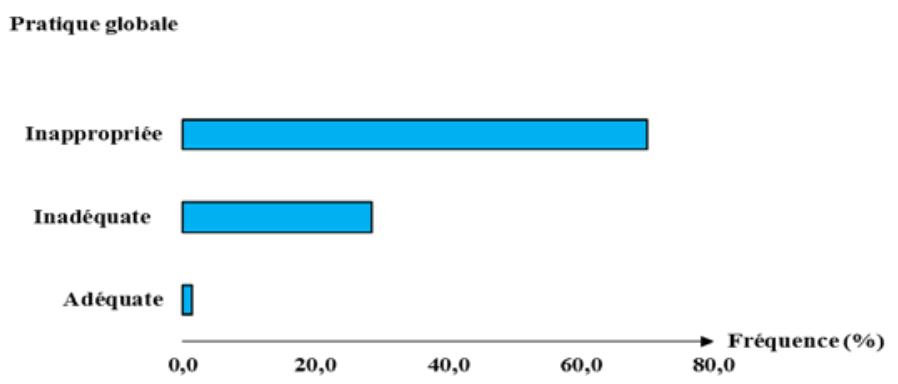

Figure 4. Répartition des agents de santé sondés sur le cancer du col selon leur pratique globale face au CCU dans les formations sanitaires publiques et privées de Parakou en 2016

$$
(\mathrm{N}=133)
$$

\section{Discussion}

Dans notre étude, 92,5\% des agents affirment avoir une bonne connaissance du cancer du col de l'utérus mais de façon objective, seuls 1,5\% l'avaient. Cet écart entre la connaissance subjective et objective pourrait s'expliquer par le défaut de pratique de dépistage et de prise en charge du cancer du col par ces agents.

Une des mesures préventives primaires du cancer du col de l'utérus repose essentiellement sur l'éviction des principaux facteurs de risque de ce cancer. Le niveau de connaissance de ces facteurs de risque est très faible $(1,5 \%)$ dans notre étude contrairement à une étude ougandaise (29\%)(Twaha 
et al,2006) . la différence des échelles de mesure du niveau de connaissance et la rareté de la formation médicale continue sur ce cancer au sein de notre population pourraient expliquer ces écarts. En effet nous avons utilisé l'échelle d'Essi et al (2013), plus récente et exhaustive contrairement à l'échelle ougandaise qui était de nature binaire. Le cancer du col de l'utérus est lié à l'infection persistante par certains papillomavirus humains (HPV) dits oncogènes (Baldauf et al, 2010). Il existe une forte corrélation entre ce cancer et le papillomavirus humain surtout les types 16, 18 et 36 (Komongui et al, 2000). En effet, 70\% de cancer du col de l'utérus sont causés par le HPV (16 et 18) (Clifford et al, 2006). C'est le principal facteur étiologique (Jeyachelvi et al,2016). Les agents de santé (39,8\%) dans notre étude ont aussi identifié le papillomavirus comme principal facteur de risque, ce qui est supérieur aux pourcentages (21\% et $29 \%$ ) observés respectivement dans une étude Marocaine et ougandaise portant uniquement sur les médecins généralistes (Nani et al,2013 ;Twaha et al,2006).Une étude Thaïlandaise, avait trouvé une fréquence nettement supérieure (82,57\%) (Songthap et al, 2009). La disponibilité du vaccin bivalent (cervarix) à Bangkok deux ans avant l'étude avait permis donc de faire la relation entre le HPV et ce cancer.

La prévention primaire du cancer du col de l'utérus repose également sur la vaccination contre le papillomavirus. L'existence de vaccin contre ce cancer est évoquée par 33,8\% des agents dans notre étude, un pourcentage plus faible $(14,4 \%)$ a été retrouvé dans une étude Nigériane portant uniquement sur les étudiantes à l'université de Lagos (Makwe et al,2012).Une étude Camerounaise a trouvé un pourcentage plus élevé (42,12\%)( McCarey et al, 2011). La vaccination contre le papillomavirus est un concept nouveau chez les agents de santé Béninois (première campagne de vaccination dans 5 communes du Bénin du 24 au 26 Mai 2016) (Sossou, 2016) et Camerounais excepté les gynécologues.

Dans notre étude on note une faible connaissance des signes évocateurs (38,3\%), des moyens de dépistage (6,8\%), des examens paracliniques (11,3\%) et des méthodes thérapeutiques (3,8\%). Des fréquences élevées ont été retrouvées par Komongui et al (2000) respectivement 95,2\% 98,4\% ; 90,3\% et $87,5 \%$ (Tonato Bagnan et al,2013). Ces variations seraient dues non seulement à l'échelle de mesure mais aussi à l'insuffisance de formation médicale continue. Pour ce qui concerne les méthodes thérapeutiques, le fait que la majorité de nos enquêtés (infirmiers (ères) et sages-femmes) ne sont pas impliqués dans la prise en charge thérapeutique, pourraient expliquer ce faible taux.

Le niveau de connaissance globale des agents sur le cancer du col de l'utérus est moyen $(71,4 \%)$ dans notre étude. Le même constat a été fais dans une étude thaïlandaise (Songthap et al, 2009). Par contre 90\% des étudiants en médecine Malaisiens dans l'étude de Mari Kannan avaient une connaissance 
élevée (Maharajan et al,2015). La différence d'échelle d'évaluation, la disponibilité du vaccin contre ce cancer en Thaïlande (4 ans avant l'étude) et les notions récentes reçues et bien assimilées pourraient expliquer cette différence

Dans notre étude, 98,5\% des agents perçoivent le cancer du col de l'utérus comme étant une affection grave. Ce résultat concorde avec celui d'une étude Malaisienne chez les étudiants en médecine 92,7\% (Maharajan et $\mathrm{al}, 2015)$.

Pour 92,5\% des agents dans notre série, le cancer du col de l'utérus est curable à condition que le diagnostic soit précoce et la prise en charge adéquate. Des pourcentages aussi élevés (81,6\%), (81\%) avaient été retrouvés respectivement par une étude Thaïlandaise et ougandaise (Twaha et al, 2006 ; Oranratanaphan et al , 2010).

Au Bénin, il existe au ministère de la santé un Programme National de Lutte contre les Maladies Non Transmissibles. Ce programme s'est fixé comme objectif pour ce qui est du cancer du col de l'utérus de réduire sa prévalence. Des stratégies et activités ont été élaborées dans ce sens. Parmi ces stratégies figuraient la mise en place de deux registres de cancer (à Cotonou et à Parakou), la mise en place d'un programme de dépistage du cancer du col utérin et la formation de 50 médecins et 150 agents au dépistage du cancer du col de l'utérus. Le faible financement actuel du programme (principale source : budget national) ne permet pas le fonctionnement des deux registres de cancer et les autres stratégies (Ministère de la santé, 2007).On comprend alors que $86,5 \%$ des agents participant à notre étude affirment que le dépistage du cancer du col de l'utérus est mal organisé ou inexistant au Bénin. Notons qu'un pourcentage non négligeable $(9,8 \%)$ dans notre série affirme n'avoir aucune information sur la conduite du dépistage de ce cancer au Bénin.

Le cancer du col présente l'intérêt d'être accessible au dépistage et 92,5\% des agents ont reconnu que la détection précoce des lésions précancéreuses et la prise en charge adéquate conduisent à la guérison. Il existe deux types de dépistage. Il s'agit de dépistage organisé et d'opportunité (OMS, 2007). Selon l'OMS, le dépistage organisé mis en place dans certains pays de l'Europe du Nord a permis de réduire de $80 \%$ l'incidence du cancer du col de l'utérus (OMS, 2008). Au Bénin, il n’existe pas de dépistage organisé de cette pathologie, le dépistage qui est individuel chez nous, n'est pas encore suffisamment ancré dans l'habitude des femmes. Pour preuve, parmi les 106 agents de santé de sexe féminin de notre étude, seules 35,8\% ont bénéficié une fois du FCV. C'est avec la cytologie que l'on possède l'expérience la plus complète et la plus longue (depuis 1950) en matière de dépistage du cancer du col de l'utérus et ceci du fait de son efficacité à réduire l'incidence de cette pathologie et donc la mortalité associée (OMS, 2007). Cette technique a 
beaucoup d'exigences et est onéreuse contrairement aux méthodes visuelles. Ces exigences rendent la cytologie peu accessible aux femmes dans les pays en développement aux ressources limitées. On comprend donc que malgré son efficacité, d'ailleurs reconnue par nos enquêtés $(77,4 \%)$ seulement $63,2 \%$ soient favorables à la pratique systématique du FCV alors que $85,7 \%$ sont favorables à la pratique systématique de IVA/IVL lors des consultations. La proportion des agents favorables au dépistage systématique par le FCV dans notre série est inférieure à celle de Komongui et al. qui avaient trouvé une proportion de $89 \%$ (2000). Cette différence pourrait s'expliquer par le fait que leur étude a porté en majorité sur des centres de gynécologie et d'obstétrique comme le témoigne la grande proportion des gynécologues obstétriciens $21 \%$ contre 3,8\% dans notre étude.

Sur les 133 enquêtés, 105 (78,9\%) pratiquent le dépistage du cancer du col de l'utérus chez les patientes alors que, seulement 83 (62,4\%) agents de santé ont reçu une formation initiale sur le dépistage. Ces agents n’ayant pas bénéficié d'une formation formelle ont dû apprendre auprès des collègues. Ceci montre la nécessité d'une formation médicale continue sur le dépistage du cancer du col de l'utérus. En ce qui concerne les méthodes de dépistage, on constate que les méthodes visuelles $(71,4 \%)$ sont plus utilisées que le frottis cervico vaginal (14,3\%).En effet, les méthodes visuelles sont des techniques relativement simples, qui donnent des résultats immédiats et constituent des approches moins onéreuses contrairement au FCV qui nécessite un personnel qualifié, des laboratoires bien équipés et un moyen de transport des échantillons efficace (OMS, 2007).

Parlant de pratique personnel du dépistage, sur les 106 agents de sexe féminin, seulement 38 soit 35,8\% ont bénéficié elles-mêmes une fois du FCV. Cette fréquence est nettement supérieure à celle d'une étude Nigériane (5,8\%) portant uniquement sur les infirmières et à celle d'une étude au Sri Lanka $(17,2 \%)$ portant uniquement sur les agents de santé de sexe féminin (Udigwe, 2006 ; Nilaweera et al, 2012). Certains auteurs ont rapporté comme raisons l'absence de signes cliniques, , la négligence et la peur .(Nani et al, 2013 ; Udigwe, 2006 ; Nilaweera et al, 2012.

\section{Conclusion}

La connaissance, l'attitude et la pratique du personnel de notre population restent faible. Ces résultats mettent en évidence la nécessité d'une formation continue des professionnels de santé y compris les paramédicaux en matière du cancer du col de l'utérus. 


\section{References:}

1. Baldauf JJ,Bergeron C, Bernigand E, Clerg S, Pinieux I, Saint-Jean M (2010) Etat des lieux et recommandations pour le dépistage du cancer du col de l'utérus en France. (HAS) 2010 ;235 :17-8.

2. Clifford G, Franceschi S, Diaz M, Munoz N, Villa LL(2006). HPV type-distribution in women with and without cervical neoplastic diseases. Vaccine ; 24 (3): 26-34.

3. Essi MJ, Njoya O (2013) L'Enquête CAP (Connaissances, Attitudes, Pratiques) en Recherche Médicale. Health Sci. Dis ; 14 (2) :1-3.

4. Isautier S(2012). Place de la vaccination antipapillomavirus humains dans la prévention du cancer du col de l'utérus.L'ile de Reunion : Univ-Lorraine : 220p.

5. Jeyachelvi K , Juwita S , Norwati D.(2016) Human papillomavirus Infection and its Vaccines: Knowledge and Attitudes of Primary Health Clinic Nurses in Kelantan, Malaysia. Asian Pac J Cancer Prev ; 17 (8), 3983-8.

6. Komongui D.G., adisso. S., Lokossou A., Yacoubou. A., Perrin X.R. et Alihonou M. (2000). Connaissances, attitudes et pratiques en matière de cancer du sein et du col de l'utérus.Abstrat congrès SAGO Cotonou 2000 ;page 60-70.

7. Lansac J, Lecomte P, Marret H (2007). Investigation en gynécologie, cancer du col de l'utérus: Collection pour le praticien gynécologie.Ed : 7; Paris : Masson; 2007 : 21-93.

8. Maharajan, M. K., Rajiah, K., Num, K. S. F., \& Yong, N. J (2015). Knowledge of Human Papillomavirus Infection, Cervical Cancer and Willingness to pay for Cervical Cancer Vaccination among Ethnically Diverse Medical Students in Malaysia. Asian Pac J Cancer Prev, 16 (14), 5733-9.

9. Makwe, C. C., Anorlu, R. I., \& Odeyemi, K. A.(2012). Human papillomavirus (HPV) infection and vaccines: knowledge, attitude and perception among female students at the University of Lagos, Lagos, Nigeria. Journal of epidemiology and global health, 2(4), 199-206.

10. . McCarey C, Pirek D, Tebeu PM, Boulvain M, Doh SA, Petignat P (2011). Awareness of HPV and cervical cancer prevention among Cameroonian healthcare workers. BMC Women ' s Health, 11(1), 45.

11. Ministère de la santé (Bénin)(2007). Plan stratégique intégré de lutte contre les maladies non transmissibles 2014-2018.Cotonou:MS : PNLMNT;2007.

12. Nani S, Benallal M, Hassoune S, Kissi D, Maaroufi A(2013). Participation des médecins généralistes de la province de Benimellal (maroc). PAMJ; 17:152. 
13. .Nilaweera, R. I. W., Perera, S., Paranagama, N., \& Anushyanthan, A. S. (2012). Knowledge and practices on breast and cervical cancer screening methods among female health care workers: a Sri Lankan experience. Asian Pacific Journal of Cancer Prevention, 13(4), 11931196.

14. Oranratanaphan S, Amatyakul P, Iramaneerat K, Srithipayawan S (2010). Knowledge, attitudes and practices about the Pap smear among medical workers in Naresuan University Hospital, Thailand. Asian Pac J Cancer Prev.;11(6):1727-30.

15. Organisation Mondiale de la Santé (Genève) (2008). Incidence et mortalité estimées du cancer du col de l'utérus dans les régions Africaines de l'OMS (tous âges confondus) en 2008.Globacan : OMS : IARC ; 2008.

16. Organisation Mondiale de la Santé (Genève(2007). La lutte contre le cancer du col de l'utérus : Guide des pratiques essentielles dans Prise en charge intégrée de la santé génésique et sexuelle et des maladies chroniques. Genève: OMS; 2007. 300p.

17. .Songthap A., Pitisuttithum P., Kaewkungwal J., Fungladda W., Bussaratid V., \& Koonsaeng, (2009) S Knowledge, attitudes, and acceptability of a human papillomavirus vaccine among healthcare providers in hospitals located in Bangkok, Thailand. southeast asian $\mathrm{j}$ trop med public health ; 40(5) :1048-56.

18. Sossou RA. (2016) Le cancer du col de l'utérus : « Le taux de guérison du cancer du col de l'utérus est très limité ». Qotidien Fraternité Doss Press 20160526.

19. Tonato Bagnan JA,Dénakpo JL, Agnida B, Hounkpatin L , Lokossou A , De Souza J (2013). Épidémiologie des cancers gynécologiques et mammaires à l'hôpital de la Mère et de l'Enfant-Lagune (HOMEL) et à la clinique universitaire de gynécologie et d'obstétrique (CUGO) de Cotonou, Bénin. Bulletin du Cancer ; 100(2) : 141-6.

20. Twaha M, Mmiro FA, Weiderpass E. (2006) Knowledge, attitudes and practices on cervical cancer screening among the medical workers of Mulago Hospital, Uganda. BMC Medical Education 6(13) :1-4.

21. Udigwe, G. O. (2006). Knowledge, attitude and practice of cervical cancer screening (pap smear) among female nurses in Nnewi, South Eastern Nigeria. Nigerian journal of clinical practice, 9(1), 40-43. 